\section{P2-135 ANTROPOMETRIC COMPARISONS IN TYPE 2 DIABETES PATIENTS, RELATED TO POPULATIONS WITH DIFFERENT SOCIAL EXPERIENCE}

doi:10.1136/jech.2011.142976i.70

${ }^{1} \mathrm{M}$ Khalangot, ${ }^{*}{ }^{2} \mathrm{E}$ Strele. ${ }^{1}$ V.P. Komisarenko Institute of Endocrinology and Metabolism, National Academy of Med Sci, Kiev, Ukraine; ${ }^{2}$ Department of Epidemiology, Riga Stradins University, Riga, Latvia

According to data from five full regional diabetes registers, prevalence of type 2 diabetes (T2D) in Ukraine among persons over 39 years of age is higher for women. The two regions (cluster 1) joined the USSR after 1939 and their population was not exposed to the famine that occurred in the middle of 1930s and which was significant in other three regions (cluster 2). Height and Body Mass Index (BMI) were assessed according to individual patient data $(\mathrm{n}=94460)$. Wilcoxon test was used to compare height $(\mathrm{cm})$ and BMI $\left(\mathrm{kg} / \mathrm{m}^{2}\right)$ values in patients, born from 1925 to 1955 in cluster $1(n=16550)$ and cluster $2(n=46853)$. In cluster 2 , the height for women was lower than in cluster 1: 162.37 (162.31-162.42) and $163.13(163.04-163.23) \mathrm{cm}$ respectively, mean (95\% CI), $\mathrm{p}<0.001$, whereas BMI was higher: 29.13 (29.09 to 29.17) and 28.64 (28.57 to 28.71$) \mathrm{kg} / \mathrm{m}^{2}, \mathrm{p}<0.001$. Height for men did not differ: 171.75 (171.66 to 171.84 ) and $171.83(171.7$ to 171.95$) \mathrm{cm}$ in cluster 2 and 1, BMI was higher: 27.83 (27.77 to 27.88) and 27.64 (27.56 to 27.72$) \mathrm{kg} / \mathrm{m}^{2}$ in cluster 2 and 1 respectively, $\mathrm{p}=0.007$. However, out of 31 yearly birth groups (YBGs) this was true for 23 YBGs in females and only for 4 YBGs in males. Height and BMI variations in $\mathrm{T} 2 \mathrm{D}$ populations affect mainly women. The reason for differences between clusters could be the result of the genotype selection due to better survival of overweight persons under the condition of starvation. "Thrifty genotype" contamination can be one of the reasons of higher T2D prevalence in Ukrainian women population.

\section{P2-136 FAMILY HISTORY OF DIABETES MODIFIES THE EFFECT OF BLOOD PRESSURE FOR INCIDENT DIABETES IN MIDDLE EASTERN WOMEN: TEHRAN LIPID AND GLUCOSE STUDY}

\author{
doi:10.1136/jech.2011.142976i.71
}

${ }^{1} \mathrm{M}$ Hatami, ${ }^{1} \mathrm{~F}$ Hadaegh, ${ }^{1,2} \mathrm{D}$ Khalili, ${ }^{1} \mathrm{~F}$ Sheikholeslami, ${ }^{1} \mathrm{~F}$ Azizi. ${ }^{1}$ Prevention of Metabolic Disorders Research Center, Research Institute for Endocrine Sciences, Shahid Beheshti University of Medical Sciences, Tehran, Iran; ${ }^{2}$ Department of Epidemiology, School of public health, Shahid Beheshti University of Medical Sciences, Tehran, Iran

Introduction Elevated blood pressure may lead to incident diabetes. Yet, data about the effect of different blood pressure components on incident diabetes in Middle Eastern women is lacking.

Methods we evaluated systolic blood pressure (SBP), diastolic blood pressure (DBP), pulse pressure (PP) and mean arterial pressure (MAP) as independent predictors of diabetes in Iranian women. We performed a population-based prospective study among 3028 nondiabetic women, aged $\geq 20$ years. ORs of diabetes were calculated for every 1 SD increase in SBP, DBP, PP and MAP.

Results During $\approx 6$ years of follow-up, 220 women developed diabetes. There were significant interactions between family history of diabetes and SBP, PP and MAP $(p \leq 0.01)$ in predicting incident diabetes. In women without a family history of diabetes, all blood pressure components were significantly associated with diabetes in the age adjusted model; the risk-factor-adjusted ORs were significant $(\mathrm{p}<0.05)$ for SBP, PP and MAP (1.30, 1.34 and 1.27, respectively) with similar predictive ability (area under receiver operator characteristic curve $\approx 83 \%$ ). In women with family history of diabetes, in the age adjusted model, SBP, DBP and MAP were associated with diabetes; in multivariable model, they were not independent predictors of diabetes.

Conclusion In women without family history of diabetes, SBP, PP and MAP, were independent predictors of diabetes with almost similar predictive ability; hence in the evaluation of the risk of blood pressure components for prediction of diabetes, the presence of family history of diabetes should be considered.

\section{P2-137 THE ASSOCIATION BETWEEN QUALITY OF PRIMARY CARE, DEPRIVATION AND CORONARY HEART DISEASE OUTCOMES}

doi:10.1136/jech.2011.142976i.72

R Khan, ${ }^{*}$ R Maclean, D Lemon. NHS Dorset, Dorchester, UK

The Quality and Outcomes Framework (QOF) is a financial incentive scheme for general practitioners in the UK. It was introduced in 2004 with the aim of improving the quality of primary care and reducing health inequalities in primary care delivery. There is relatively little evidence to support the hypothesis that practices with higher quality of care measured by the QOF score have better health outcomes. However some studies have shown that higher QOF scores are associated with lower admission (Bottle et al. 2008b; Kiran et al. 2010). In both studies the association was stronger in practices with higher deprivation/lower socioeconomic distribution. The aim of this study is to assess whether high quality primary care, measured using quality of care measures, is associated with lower coronary heart disease morbidity and mortality in an area of relatively low deprivation. This is an ecological study using data from four years before and four years after the introduction of QOF. The study will compare these time periods for any change in CHD admissions and mortality. The study will also assess whether there is an association between quality of care measures on specific coronary heart disease and associated hospital admission rates and mortality rates. The study will also assess whether any association is affected by the practice based deprivation score. Results of this study will be presented at the conference.

\section{P2-138 PARENTAL INFLUENCES ON CARDIOVASCULAR RISK- FACTORS IN SWEDISH CHILDREN}

doi:10.1136/jech.2011.142976i.73

${ }^{1}$ A Khanolkar, ${ }^{*}{ }^{1}$ I Koupil, ${ }^{2}$ L Byberg. ${ }^{1}$ Centre for Health Equity Studies (CHESS), Karolinska Institutet/Stockholm University, Stockholm, Sweden; ${ }^{2} U p p s a l a$ Clinical Research Center and Department of Surgical Sciences, Section of Orthopaedics, Uppsala University, Uppsala, Sweden

Background Precursors of cardiovascular diseases (CVD) originate in childhood. We investigated the relationship of children's CVD riskfactors with parent's socioeconomic position (SEP) and lifestyle. We also studied how CVD risk-factors correlate within families.

Methods We studied 602 families (2141 individuals) comprising two full sibs; aged 5-14 years, and their biological parents (Uppsala Family Study). Parental SEP measured as occupational class and education, and lifestyle habits (smoking, physical activity, alcohol consumption) were obtained from questionnaires. Associations with cholesterol, apolipoproteins (ApoB/ApoA1), adiponectin, blood pressure, body mass index and overweight/obesity were analysed by linear and logistic regression. Results are adjusted for children's age, gender, pubertal stage and family clustering.

Results In addition to differences in children's CVD risk factors by parental SEP, we observed specific associations between parental lifestyle and children's risk-factors that were independent of parental social characteristics. Children of non smoking parents had 
lower BMI (difference between children of smoking vs non smoking fathers, $0.8 \mathrm{~kg} / \mathrm{m}^{2}, 95 \%$ CI 0.1 to 1.4 ) and children of mothers reporting vigorous physical activity had lower BMI, cholesterol, ApoB/ApoA1 levels and decreased odds for overweight/obesity. Independent and consistently statistically significant associations were found between parents' and children's CVD risk-factors.

Conclusion Parental behaviours like smoking, alcohol consumption, and low physical activity were significantly associated with higher levels of certain CVD risk-factors in children. Strong correlations in CVD risk-factors between family members that are not related to SEP or parental lifestyle suggest a role of genetics in influencing children's CVD risk-factors. Public health policies should target families with unhealthy lifestyles.

\section{P2-139 PERIODONTAL INFLAMMATIONS ARE RELATED TO NITRIC OXIDE LEVELS IN SALIVA}

doi:10.1136/jech.2011.142976i.74

H-D Kim, ${ }^{*}$ D-H Han, H-M Kim, H-S Shin, M-S Kim. Seoul National University, Seoul, Republic of Korea

Introduction Nitric oxide (NO) plays an important role in almost every biological system. The increase in NO in periodontal tissues has been reported in inflammatory periodontal disease, which suggests the production and participation of $\mathrm{NO}$ in the disease process. However, the association between salivary NO levels and periodontitis was rarely reported. The aim of this study was to assess the periodontal health according to the salivary NO levels in Korean elderly.

Methods 203 subjects aged 48-84 years old were cross-sectionally surveyed. All participants underwent oral examination. Age, gender, education level, regular exercise, smoking were evaluated through interview. Periodontal health status was evaluated by clinical attachment loss over $6 \mathrm{~mm}$ (CAL6 $\mathrm{mm}$ ) for 6 points of 12 index teeth (total 72 sites). The number of CAL $6 \mathrm{~mm}$ sites was counted. Unstimulated saliva was collected for $10 \mathrm{~min}$. Salivary NO was determined by the Griess reagent. ANCOVA and Multiple linear regression analyses were applied.

Results After correcting for differences in for age, gender, education, regular exercise, smoking, salivary flow rate, and number of natural teeth, high NO level group showed the highest number of CAL6 $\mathrm{mm}$ sites (15.2 vs 7.0 and 10.8, $\mathrm{p}=0.031$ ). Salivary NO levels had a dose-effect relationship with the number of CAL6 $\mathrm{mm}$ sites $(\beta=0.026, p=0.011)$.

Conclusion NO levels are elevated in individuals with periodontitis. These results reveal that periodontal disease and its severity are related to salivary $\mathrm{NO}$ concentration, indicating that $\mathrm{NO}$ may serve as a potential biological marker for detection and monitoring of periodontitis.

\section{P2-140 DIETARY PATTERNS AND RISK OF METABOLIC SYNDROME AMONG KOREAN POPULATION: THE KOREAN NATIONAL HEALTH AND NUTRITION EXAMINATION SURVEY 2007-2009}

doi:10.1136/jech.2011.142976i.75

${ }^{1} \mathrm{M} \mathrm{K} \mathrm{Kim},{ }^{*}{ }^{2} \mathrm{M}$ Lee, ${ }^{3} \mathrm{C}$ Shin, ${ }^{4} \mathrm{I}$ Baik, ${ }^{1} \mathrm{Y}$ Yun, ${ }^{5} \mathrm{H}$ Okubo, ${ }^{5} \mathrm{~S}$ Sasaki. ${ }^{1}$ Cancer Epidemiology Branch, National Cancer Center, Goyang, Republic of Korea; ${ }^{2}$ Department of Food and Nutrition and Research Institute of Obesity Sciences, Sungshin Women's University, Seoul, Republic of Korea; ${ }^{3}$ Department of Internal Medicine, Korea University Ansan Hospital, Ansan, Republic of Korea; ${ }^{4}$ Department of Foods and Nutrition, College of Natural Sciences, Kookmin University, Seoul, Republic of Korea;
${ }^{5}$ Department of Social and Preventive Epidemiology, Graduate School of Medicine, the University of Tokyo, Tokyo, Japan

An increasing number of studies in Western countries have examined the relationship between dietary pattern, namely the measurement of overall diet by considering the cumulative effects of nutrients, and the risk of metabolic syndrome (MetS). However, such information is absolutely lacking among Asian populations, including Korea, with different subject characteristics and culturespecific dietary habits. This cross-sectional study examined the association between dietary pattern identified by factor analysis and the risk of MetS among Korean population. We used data from the Korean National Health and Nutrition Examination Survey, 2007-2009. The analytical sample included men and women aged 30-80 years with $24 \mathrm{~h}$ recall, anthropometric and clinical measurements $(n=5320)$. MetS was defined based on the Adult Treatment Panel III of the National Cholesterol Education Program criteria as having three or more risk factors using a modified obesity index. We identified three dietary patterns ("Meat \& alcohol", "Unbalanced Korean", and "Diverse") in both sexes. After adjustment for potential confounding factors, subjects in the highest quintile of the "Diverse" dietary pattern had significantly lower risk of MetS compared with those in the lowest in both sexes (multivariate OR: $0.50 ; 95 \% \mathrm{CI}$ 0.27 to 0.90 ; $p$ for trend $=0.174$ for men; multivariate OR: $0.58 ; 95 \%$ CI 0.38 to 0.87 ; $p$ for trend $=0.005$ for women). Other dietary patterns were not associated with the risk of MetS. The result suggests that a diet high in vegetables, fruits, eggs, fish, and meat might reduce the risk of MetS among Korean population.

\section{P2-141 DEFINITION AND VALIDATION OF AN ALGORITHM TO IDENTIFY COPD PATIENTS FROM ADMINISTRATIVE DATABASES}

doi:10.1136/jech.2011.142976i.76

L Bauleo, U Kirchmayer,* V Belleudi, N Agabiti, L Pinnarelli, S Cascini, D Fusco, M Arcà, M Davoli. Department of Epidemiology of the Regional Health Service, Lazio Region, Italy

Introduction Administrative databases are increasingly used to identify patients with chronic conditions, however the optimal methodology for Chronic Obstructive Pulmonary Disease (COPD) is still debated.

Objective To develop and validate an algorithm to identify patients with COPD in Lazio (2625102 residents over 45) linking clinical and administrative data.

Methods From the regional hospitalisation, drug prescription and outpatient registries, through record linkage, we identified patterns of specific drug use (minimum 2 prescription during 12 months) and COPD hospitalisations during a 9-year period in 428 patients with COPD, who attended an outpatient clinic in 2006, and in 2140 people without COPD. Through a Bootstrap-Stepwise procedure we selected COPD associated factors. We validated the algorithm through internal (cross-validation-bootstrap, jack-knife) and external validation (comparison with external COPD patients with confirmed diagnosis).

Results A total of 205611 (7.8\%) COPD patients were identified. Factors associated with COPD were: prescription of $\beta 2$-agonists, anticholinergics, corticosteroids, oxygen, and previous hospitalisation for COPD and respiratory failure. For each patient we estimated an expected probability to suffer from COPD. Depending on the cut-point of expected probability, sensibility (SE) ranged from 0.15 to 0.87 and specificity (SP) from 0.79 to 0.99 . We defined a cutpoint of $0.30(\mathrm{SE}=64 \%$; $\mathrm{SP}=97 \%$ ) to identify the COPD patients. Applying our algorithm on external COPD patients we succeeded to identify $86 \%$ 